\title{
STAT1 promotes megakaryopoiesis downstream of GATA-1 in mice
}

\author{
Zan Huang, ${ }^{1}$ Terri D. Richmond, ${ }^{2,3}$ Andrew G. Muntean, ${ }^{1}$ \\ Dwayne L. Barber, ${ }^{2,3}$ Mitchell J. Weiss, ${ }^{4}$ and John D. Crispino ${ }^{1}$

\begin{abstract}
${ }^{1}$ Department of Medicine, Division of Hematology and Oncology, Northwestern University Feinberg School of Medicine, Chicago, Illinois, USA 2Division of Stem Cell and Developmental Biology, Ontario Cancer Institute, Toronto, Ontario, Canada.

${ }^{3}$ Department of Medical Biophysics, University of Toronto, Toronto, Ontario, Canada. ${ }^{4}$ The Children's Hospital of Philadelphia, Division of Hematology, University of Pennsylvania School of Medicine, Philadelphia, Pennsylvania, USA.
\end{abstract}

\begin{abstract}
Thrombocytosis is associated with inflammation, and certain inflammatory cytokines, including IFN- $\gamma$, stimulate megakaryocyte and platelet production. However, the roles of IFN- $\gamma$ and its downstream effector STAT1 in megakaryocyte development are poorly understood. We previously reported that STAT1 expression was significantly downregulated in Gata1-knockdown murine megakaryocytes, which also have impaired terminal maturation. Here, we show that ectopic expression of STAT1, or its target effector IRF-1, rescued multiple defects in Gata1-deficient megakaryopoiesis in mice, inducing polyploidization and expression of a subset of platelet-expressing genes. Enforced expression of STAT1, IRF-1, or GATA-1 enhanced phosphorylation of STAT1, STAT3, and STAT5 in cultured Gata1-deficient murine megakaryocytes, with concomitant megakaryocyte maturation. In contrast, enhanced thrombopoietin signaling, conferred by enforced expression of constitutively active JAK2 or c-MPL, induced phosphorylation of STAT3 and STAT5, but not STAT1, and failed to rescue megakaryocyte maturation. Finally, megakaryocytes from Stat1 $1^{-/}$mice were defective in polyploidization. Together, these findings reveal a unique role for STAT1 in megakaryopoiesis and provide new insights into how GATA-1 regulates this process. Our studies elucidate potential mechanisms by which various inflammatory disorders can cause elevated platelet counts.
\end{abstract}

\section{Introduction}

Proinflammatory cytokines have a powerful impact on the hematopoietic system and can foster the expansion of the megakaryocyte lineage and subsequent thrombocytosis. Diseases such as chronic inflammation and cancer are associated with dramatically increased platelet counts, an effect that is mediated in part by aberrant cytokine signaling. Megakaryocyte progenitors receive these extracellular signals and translate them into an altered gene expression program. While the association between inflammation and thrombocytosis is well established, the specific mechanism by which inflammatory signaling affects gene expression remain unknown.

Megakaryocyte development is controlled by lineage-specific transcription factors including the zinc finger protein GATA-1 and its partner Friend of GATA-1 (FOG-1) (1). GATA-1 directs the differentiation of hematopoietic progenitors into erythrocytes and megakaryocytes and also participates in the development of eosinophils and mast cells. Mice that are deficient for Gata1 specifically in the megakaryocyte lineage (Gata1-knockdown [Gata1-KD] mice) fail to produce appropriate numbers of platelets but display a striking increase in megakaryocyte numbers $(2,3)$. Gata1-KD megakaryocytes also exhibit marked hyperproliferation both in vitro and in vivo $(3,4)$. Furthermore, Gata1-KD mice develop progressive extramedullary hematopoiesis and myelofibrosis (5). Interestingly, although mutations in GATA1 have not been found in primary myelofibrosis (PMF), megakaryocytes from individuals with PMF express reduced levels of GATA-1 (6). Thus, abnormalities of GATA-1

Nonstandard abbreviations used: $\mathrm{AMKL}$, acute megakaryocytic leukemia; KD knockdown; MPD, myeloproliferative disorder; PMF, primary myelofibrosis; SDF-1, stromal cell-derived factor 1 ; TPO, thrombopoietin.

Conflict of interest: The authors have declared that no conflict of interest exists. Citation for this article: J. Clin. Invest. 117:3890-3899 (2007). doi:10.1172/JCI33010 may contribute to the pathogenesis of PMF. Finally, GATA1 gene mutations are associated with essentially all cases of acute megakaryocytic leukemia (AMKL) in children with Down syndrome (7). Collectively, these findings highlight the requirement for GATA-1 for growth control and terminal maturation of megakaryocytes.

Numerous genes are dysregulated by the loss of Gata1 in mice $(4,8)$. Two downregulated genes, Jak2 and Stat1, mediate thrombopoietin (TPO) and IFN- $\gamma$ signaling pathways, respectively. TPO is the primary megakaryocyte-potentiating factor and is critical for normal platelet production (9). TPO functions through binding its receptor, c-Mpl, to activate JAK2, STAT1, STAT3, and STAT5 in megakaryocytes and other hematopoietic cells $(10-12)$. Moreover, hematopoietic stem cells express c-Mpl and are responsive to TPO (13).

The functions of JAK2, STAT3, and STAT5 in erythroid and megakaryocyte lineages have been extensively studied. Embryos lacking Jak2 die from severe anemia associated with deficient definitive erythropoiesis $(14,15)$. Furthermore, Jak2-deficient fetal liver myeloid progenitor cells are not responsive to erythropoietin (EPO) or TPO, demonstrating that both cytokines function through JAK2 (14). Several lines of evidence suggest that both STAT3 and STAT5 are essential for normal regulation of megakaryopoiesis. First, both of these proteins are commonly activated in megakaryoblastic leukemias and human myeloproliferative disorders (MPDs) $(16,17)$ (also see Discussion). Second, mice deficient for both STAT5A and STAT5B are thrombocytopenic, likely due to a reduction in functional hematopoietic progenitors (18). Third, transgenic expression of dominant-negative STAT3 caused a significant delay in platelet recovery after myelosuppression (19). Interestingly, a recent study showed that GATA-1 could bind and inhibit the activity of STAT3 in megakaryocytes, establishing a potential functional link between these 2 molecules (20). 
A

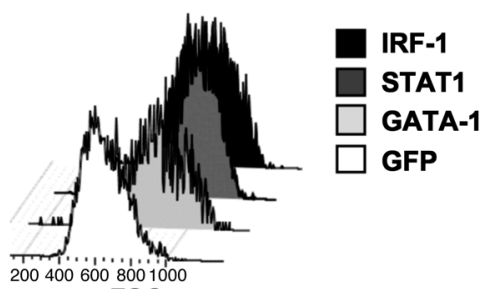

B
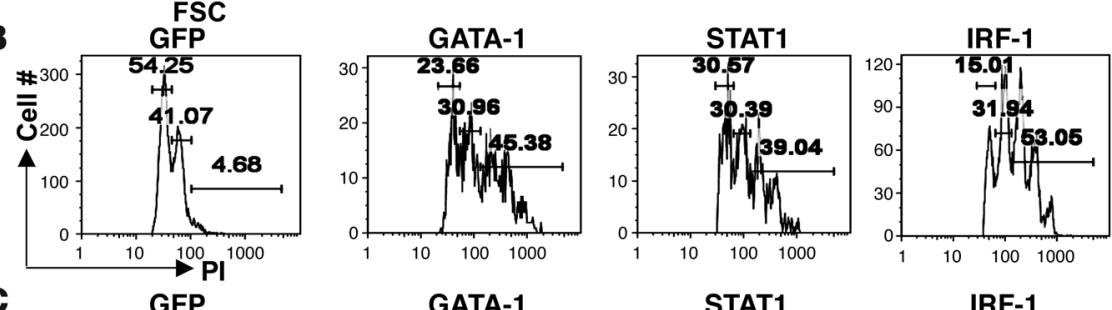

C
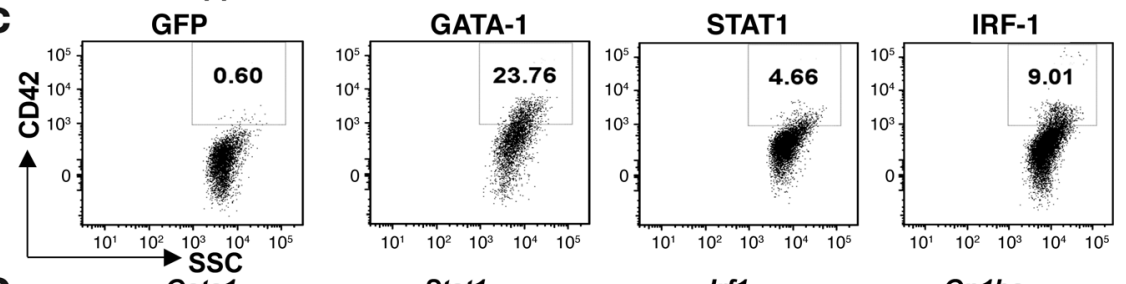

D
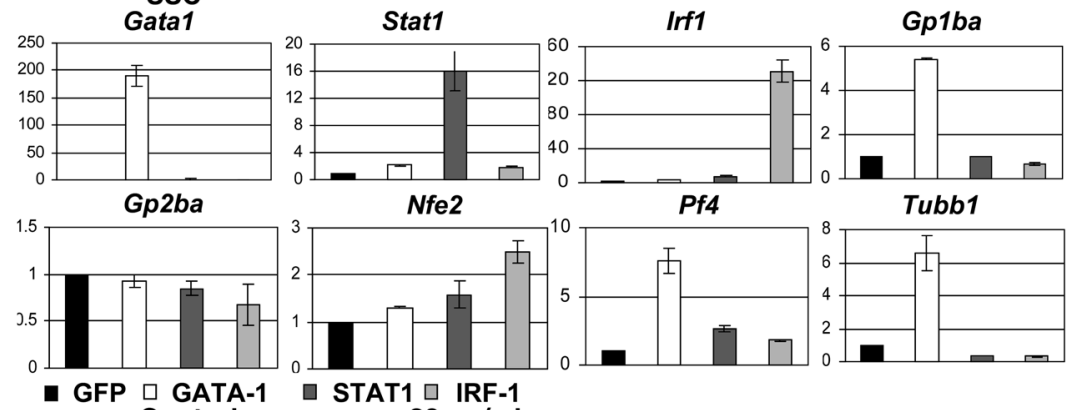

Pf4

E
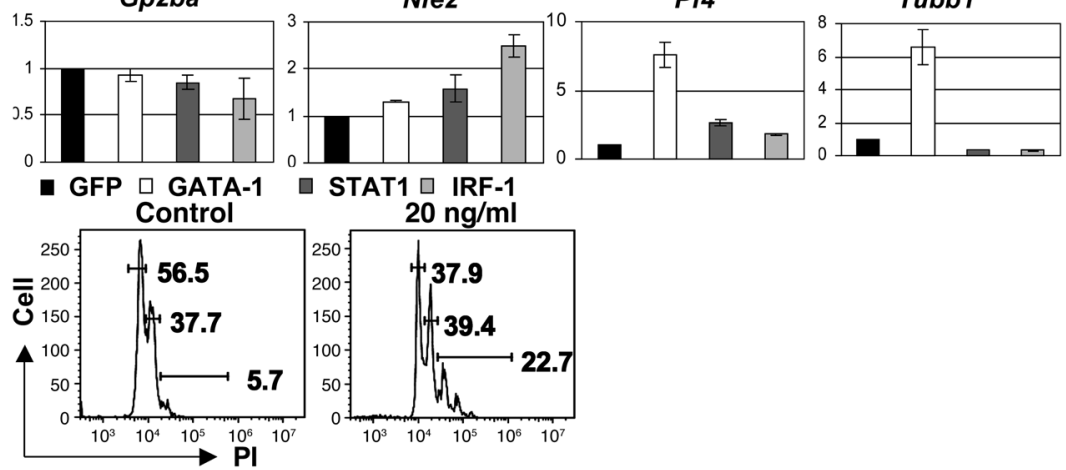

\section{Figure 1}

STAT1 signaling promotes megakaryocytic differentiation in G1ME cells. (A) G1ME cells were transduced with retroviral constructs expressing GFP alone or bicistronically expressing GFP plus GATA-1, GFP plus STAT1, or GFP plus IRF-1. Two days after infection, transduced cells were collected and megakaryocytic differentiation was assayed by flow cytometry. A gate was set on GFP+ cells. The cell size was measured with forward side scatter. (B) The polyploidy of transduced cells was analyzed by staining with propidium iodide (PI). (C) Cell-surface expression of CD42 was determined by staining with PE-labeled anti-CD42 antibody. (D) The expression of lineage-specific genes in the transduced G1ME cells was detected by quantitative RT-PCR. (E) G1ME cells were also treated with or without $20 \mathrm{ng} / \mathrm{ml}$ of IFN- $\gamma$ for 3 days. The polyploidy of treated cells was measured by staining with $\mathrm{Pl}$ and analyzed by flow cytometry. Data are representative of 3 independent experiments with similar results.
In contrast to other STAT proteins, less is known about the function of STAT1 during megakaryopoiesis. In most cell types, STAT1 is primarily triggered by interferon signaling (21). However, TPO signaling phosphorylates STAT1 in WT megakaryocytes (10). Moreover, while interferons inhibit proliferation of most hematopoietic cells, IFN- $\gamma$ promotes megakaryopoiesis both in vivo and in vitro (22-24). How IFN- $\gamma$ induces this apparently paradoxical effect on the megakaryocyte lineage is unknown. This problem has important clinical implications, since inflammatory disorders are commonly associated with increased IFN- $\gamma$, elevated platelet numbers, and thrombotic tendencies. Here, we provide insights into the mechanisms by which IFN- $\gamma$ and STAT1 promote megakaryopoiesis and platelet production by linking this signaling pathway directly to GATA-1. We show that ectopic expression of STAT1 or its downstream transcriptional target IRF-1 promotes features of megakaryocytic differentiation of G1ME cells, a Gata1-null erythromegakaryocytic cell line (25), and primary bone marrow cells derived from Gata1-KD mice. In addition, IFN- $\gamma$ itself drives polyploidization and differentiation of WT and
Gata1-KD megakaryocytes. Finally, megakaryocytes from Stat1-deficient mice exhibit reduced ploidy compared with those from littermate controls. Together, our data identify a new regulatory hierarchy through which the master regulator GATA-1 promotes megakaryopoiesis in part via activation of IFN- $\gamma /$ STAT1 signaling.

\section{Results}

Ectopic expression of STAT1 and IRF-1 promotes megakaryocytic differentiation in GATA-1-null G1ME cells. Previously, we found that STAT1 mRNA was significantly downregulated in Gata1-KD megakaryocytes (4). To examine the physiological significance of this observation, we studied the effects of ectopic STAT1 expression in G1ME cells, a Gata1-null progenitor line that undergoes erythromegakaryocytic differentiation when GATA-1 expression is restored in the presence of EPO or TPO (25). In the following experiments, we rescued G1ME cells with GATA-1-encoding retrovirus in the presence of TPO only to favor megakaryocyte production and obtained less than $2 \%$ erythroid cells. Without GATA-1, enforced STAT1 expression promoted 
A

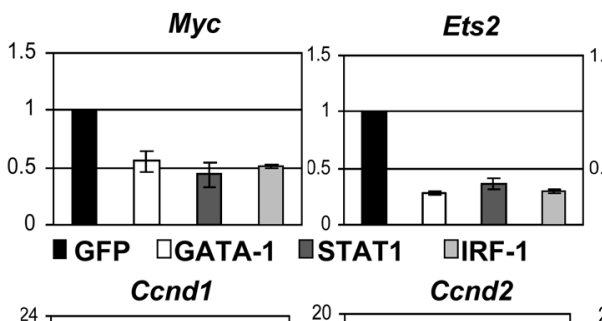

B

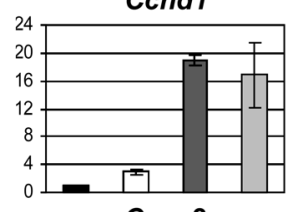

Ccne2

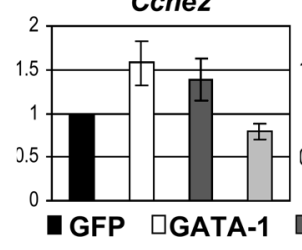

C

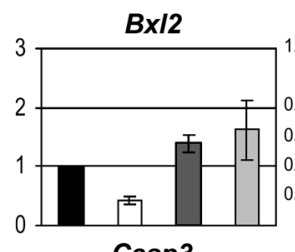

Casp3
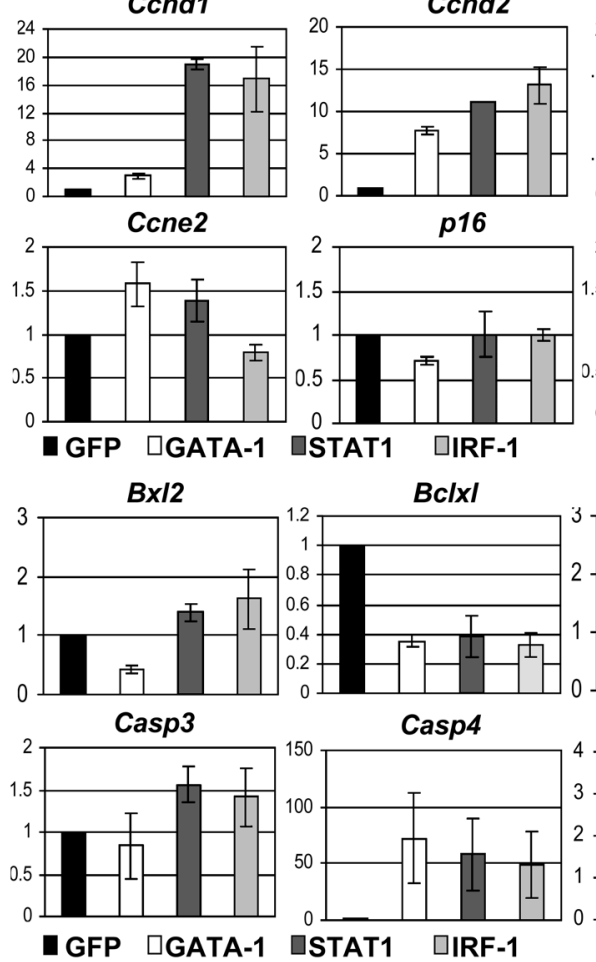

p16

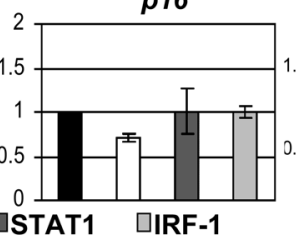

$B c \mid x I$

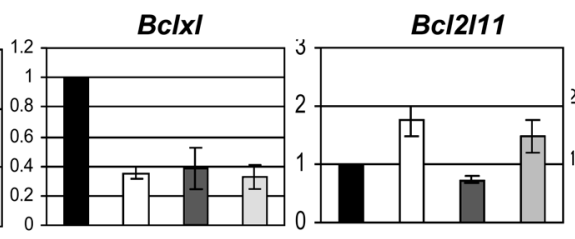

casp9
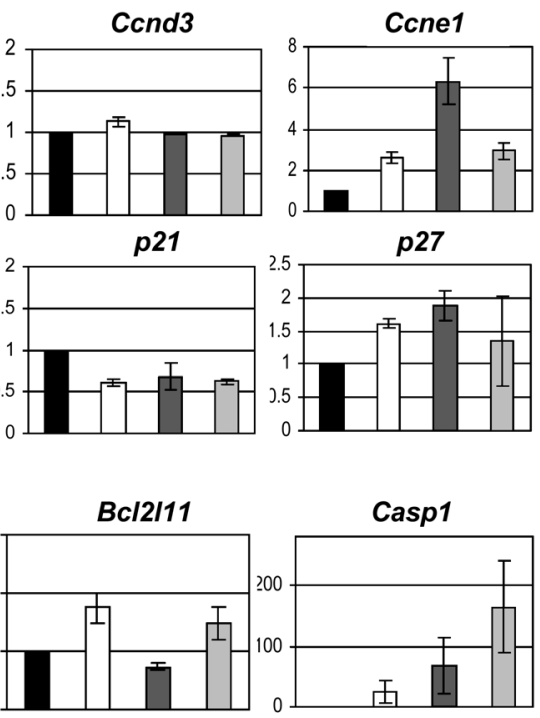

Casp12
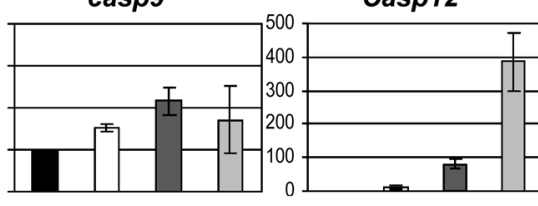

Figure 2

STAT1 and IRF-1 gene induction is a subset of GATA-1 induction in transduced G1ME cells. (A) GFP-, GATA-1-, STAT1-, or IRF-1-transduced G1ME cells were purified by sorting and cultured for an additional 2 days. Total RNA was prepared and used for quantitative RT-PCR. The expression of transcription factors was detected. (B) The expression of cell-cycle genes was also measured. (C) The expression of apoptosis genes was determined. Data are representative of 2 independent experiments performed in triplicate.

several features of megakaryocytic differentiation of G1ME cells, as evidenced by increased cell size (forward scatter) and increased DNA content, reflecting polyploidization (Figure 1, A and B). Similar effects were produced by enforced expression of IRF-1, a major STAT1 effector (reviewed in ref. 26). In addition, STAT1 or IRF-1 induced the expression of $\mathrm{CD} 42$, a late marker of megakaryocyte maturation, albeit to a much lesser extent than GATA-1 (Figure 1C). These data suggest that STAT1 signaling can bypass Gata1 deficiency to enhance multiple aspects of megakaryocytic differentiation.

To further characterize how megakaryocytic differentiation is regulated by STAT1 and IRF-1, we compared their effects to that of GATA- 1 on the expression of lineage-specific genes by quantitative RT-PCR (Figure 1D). We first measured Gata1, Stat1, and Irf1 mRNA levels in retrovirally transduced cells. GATA- 1 induced both Stat1 and Irf1 expression in G1ME cells, consistent with our previous microarray data (Figure 1D and Supplemental Table 1; supplemental material available online with this article; doi:10.1172/JCI33010DS1) (4). Moreover, GATA-1 transactivated the Stat1 promoter in lucifer- and IRF-1-transduced cells (Supplemental Figure 2A). This suggests that not all antiproliferative actions of GATA-1 are mediated through STAT1 signaling. In their final stages of maturation, megakaryocytes undergo endomitosis, shed platelets, and eventually undergo apoptosis (27). Flow cytometry revealed that 43.9\% of GATA-1-transduced cells were positive for annexin V staining compared with $6.25 \%$ of control vector-transduced cells. In contrast, $19.2 \%$ of STAT1-transduced cells and $28 \%$ of IRF-1-transduced cells were undergoing apoptosis. This is consistent with earlier findings that STAT1 signaling promotes megakaryocyte maturation but, unlike GATA-1, not to the full extent (Supplemental Figure 2B).

Altered gene expression in G1ME cells undergoing megakaryocytic differentiation. To identify STAT1 target genes that promote megakaryocyte differentiation, we performed more extensive gene expression analysis on transduced G1ME cells (Figure 2 and Supplemental Table 1). STAT1 altered the expression of many transcription factors that are known to regulate megakaryopoiesis. For example, $c-M y c$ and $E t s 2$, whose transcription is elevated in the absence of GATA-1, were downregulated by STAT1 $(4,8)$. In contrast, Runx1, 
A
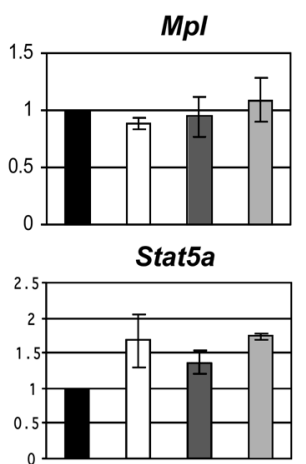

c

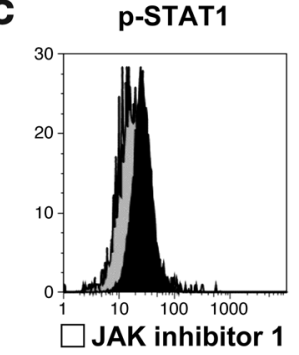

E

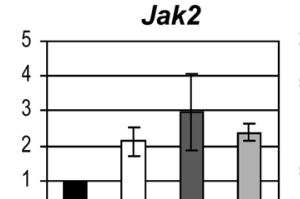

0
2.5
2
1.5
1
0.5
0

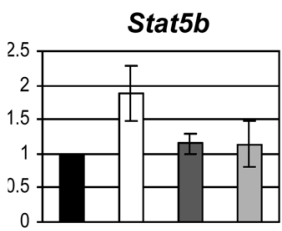

p-STAT3

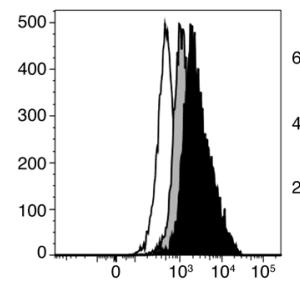

$\square$ TPO $\square$ TPO+IFN- $\gamma$

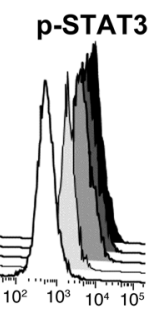

$\square$ GFP

EIRF-1

$\square$ GATA-1

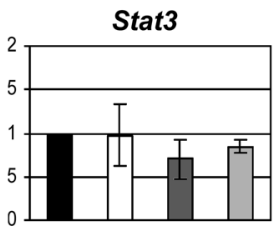

Ifngr1

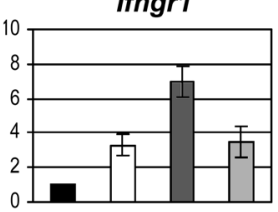

p-STAT5

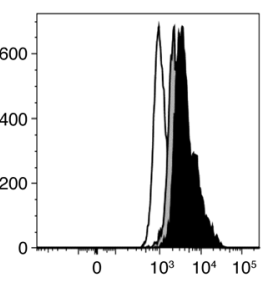

p-STAT5

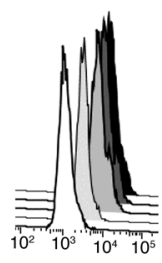

\section{$\square$ STAT1}

\section{Figure 3}

Phosphorylation of STATs is increased by GATA-1, STAT1, or IRF-1. (A) GFP-, GATA-1-, STAT1-, or IRF-1-transduced G1ME cells were purified by sorting GFP+ cells and cultured for an additional 2 days. Total RNA was prepared from sorted cells and used for quantitative RT-PCR. (B) Control cells (TPO alone; Con) or cells treated with IFN- $\gamma(20 \mathrm{ng} / \mathrm{ml})$ or JAK inhibitor 1 (Inh) $(2 \mu \mathrm{M})$ overnight were lysed in RIPA buffer. Phosphorylation of STAT1, STAT3, or STAT5 was analyzed by Western blot. (C) The cells were fixed, permeabilized, and stained with allophycocyanin- (APC-) or PE-labeled antibodies specific for phosphorylation of STAT1, STAT3, or STAT5. Phosphorylation of STAT1, STAT3, or STAT5 was analyzed by flow cytometry. (D) GFP-, GATA-1-, STAT1-, or IRF-1-transduced cells were purified by sorting. Phosphorylation of STAT1, STAT3, or STAT5 was measured by Western blot analysis in purified cells. (E) Phosphorylation of STAT1, STAT3, or STAT5 in GFP-, GATA-1-, STAT1-, or IRF-1transduced cells was detected by intracellular staining with APC- or PE-labeled antibodies specific for phosphorylation of STAT1, STAT3, or STAT5 as described above. A gate was set on GFP+ ${ }^{+}$cells. Data are representative of 3 independent experiments with similar results.

which is required for megakaryocyte maturation and cooperates with GATA-1 in megakaryocyte differentiation $(28,29)$, was upregulated by STAT1. STAT1 also induced cell-cycle genes, including Ccnd1, Ccnd2, and Ccne2 (Figure 2B), that are known to promote endomitosis (30-33).

Other signature features of megakaryocytic maturation include formation of demarcation membranes, long pseudopod extensions indicative of proplatelet formation, and eventual apoptosis. In G1ME cells, enforced GATA-1 repressed the expression of antiapoptotic genes $B c l 2$ and $B c l x l$ and induced some proapoptotic genes including Bcl2l11 (Bcl2-like factor 11) and caspase-1, -4, -9, and -12 (Figure 2C). These findings are consistent with previous reports showing that expression of antiapoptotic members of the $\mathrm{Bcl} 2$ family impaired thrombopoiesis, whereas BCL2L11 and caspases were required for proplatelet formation and platelet release (34-36). STAT1 induced the same caspase genes but had little effect on the $\mathrm{Bcl} 2$ family pro- teins. These findings indicate that subsets of GATA-1 actions in megakaryocytes are mediated through STAT1 signaling.

Enhanced TPO signaling in G1ME cells undergoing megakaryocytic differentiation. TPO, which is required for survival and proliferation of G1ME cells, functions primarily by activating JAK2 and downstream effectors STAT3, STAT5, and perhaps STAT1. In G1ME cells, ectopic GATA-1, STAT1, or IRF-1 all induced Jak2 mRNA expression (Figure 3A and Supplemental Table 1), which is significantly downregulated in Gata1-KD megakaryocytes (4). GATA-1 induced the expression of Stat1 (Figure 1D) and, to a lesser degree, Stat $5 b$ and Stat $5 a$, but did not change c-Mpl or Stat3 (Figure 3A).

Next, we examined the effects of cytokine signaling on STAT protein activation in G1ME cells by Western blot analysis and flow cytometry. STAT3 and STAT5, but not STAT1, were phosphorylated in cells cultured with TPO. In marked contrast, G1ME cells grown with both IFN- $\gamma$ and TPO showed enhanced phosphorylation 
A
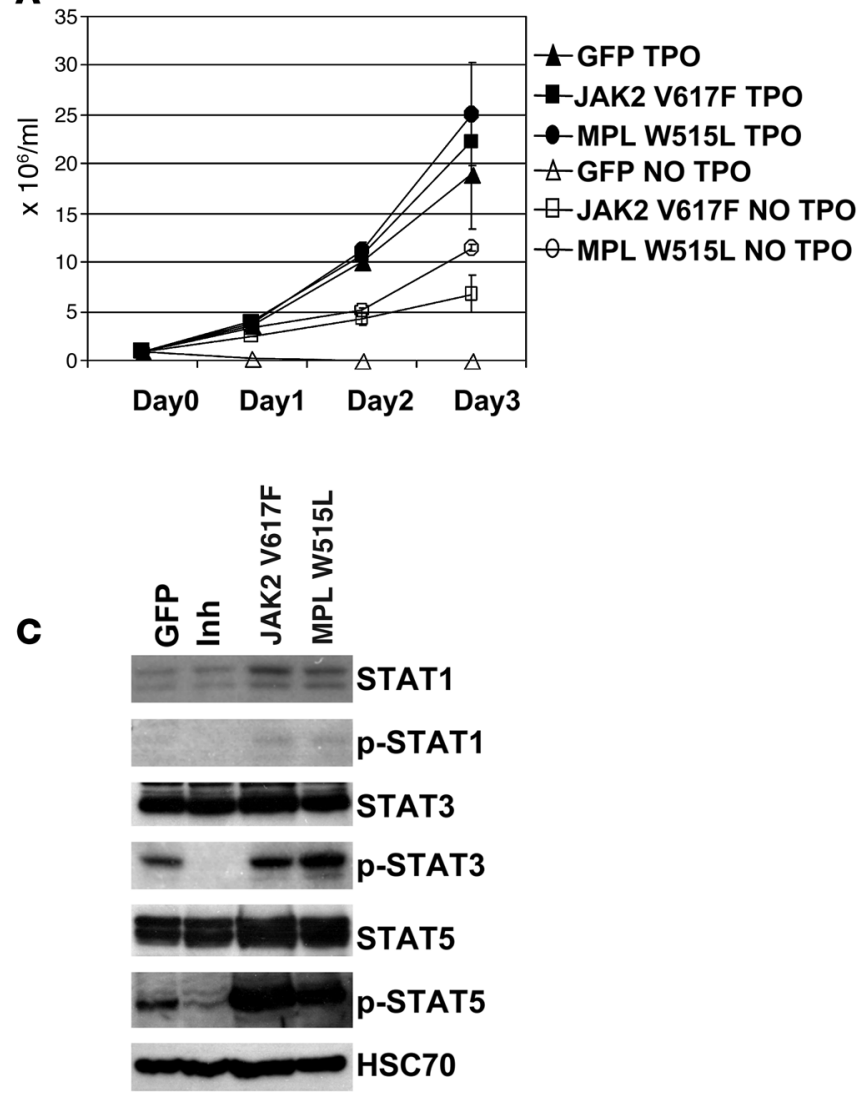

B

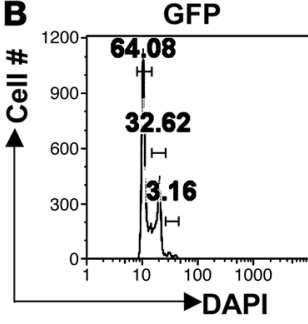

JAK2 V617F

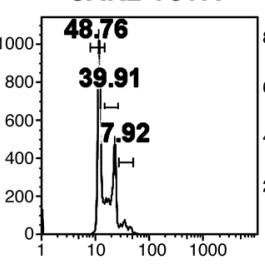

MPL W515L
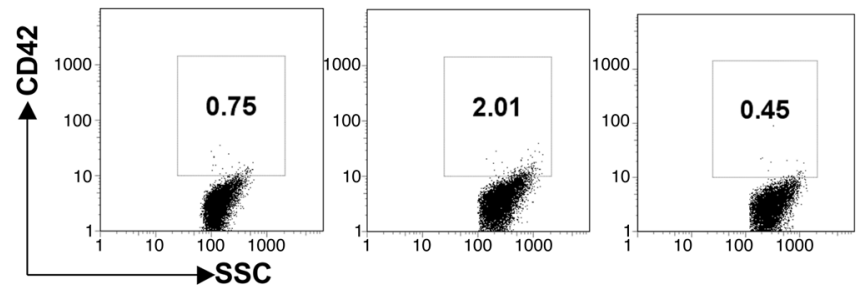

D
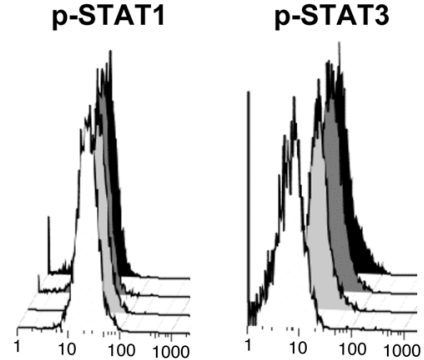

p-STAT5

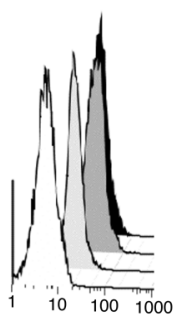

$\square$ JAK inhibitor $1 \square$ GFP JAK2 V617F

MPL W515L

Figure 4

Enhanced phosphorylation of STAT3 and STAT5 is not sufficient to promote polyploidization in G1ME cells. (A) G1ME cells transduced with GFP, JAK2 V617F, or MPL W5151L were purified by sorting and expanded. Cells were seeded at $1 \times 106 / \mathrm{ml}$ with or without $1 \%$ TPO condition media. The cell number was counted over time until day 3. Results are representative of 2 independent experiments performed in duplicate. (B) The polyploidy in GFP-, JAK2 V617F-, or MPL W515L-transduced G1ME cells was measured by staining with DAPI, and the expression of CD42 was detected by staining with PE-labeled anti-CD42 antibody and analyzed by flow cytometry. (C) Phosphorylation of STAT1, STAT3, or STAT5 in GFP-, JAK2 V617F-, or MPL W515L-transduced G1ME cells was measured by Western blot analysis. (D) Phosphorylation was also detected by intracellular staining with APC- or PE-labeled antibodies specific for phosphorylation of STAT1, STAT3, or STAT5 and analyzed by flow cytometry.

of all 3 STAT factors (Figure 3, B and C). Since upregulation of Jak2 mRNA itself might explain the enhanced phosphorylation of STAT1, STAT3, and STAT5, we measured the phosphorylation states of these molecules in the transduced G1ME cells. Enforced expression of GATA-1, STAT1, and IRF-1 all enhanced phosphorylation of STAT1, STAT3, and STAT5, coincident with megakaryocytic differentiation (Figure 3, D and E). Furthermore, we also observed impaired TPO signaling in Gata1-KD megakaryocytes derived from bone marrow cells, which show defective terminal differentiation (Supplemental Figure 3). These results suggest that phosphorylation of all 3 STAT proteins normally occurs in GATA-1mediated megakaryocyte differentiation.

Enhanced STAT phosphorylation in GATA-1-, STAT1-, or IRF-1transduced G1ME cells might be due to autocrine or paracrine effects of IFN- $\gamma$. To investigate this possibility, we evaluated IFN- $\gamma$ and its receptor Ifngr 1 expression in transduced G1ME cells. Interestingly, enforced expression of GATA-1, STAT1, or IRF-1 induced Ifngrl expression, but we did not detect Ifng expression under any conditions. Hence, IFN- $\gamma$ signaling does not contribute to the enhanced phosphorylation of STATs in G1ME cells (Figure 3A and data not shown).
Enhanced phosphorylation of STAT5 and STAT3 is not sufficient to promote megakaryocytic differentiation in G1ME cells. Both STAT1 and GATA-1 enhance TPO signaling and promote megakaryocytic maturation. To test whether enhanced TPO signaling is sufficient to induce megakaryocytic differentiation in G1ME cells, we tested the effects of constitutively active forms of JAK2 (V617F) or c-MPL (W515L). Mutations in JAK2 and $c-M P L$ are uniquely associated with human MPDs, including PMF (reviewed in ref. 37). Both JAK2 V617F and c-Mpl W515L conferred TPO-independent growth but did not promote megakaryocytic maturation, as evidenced by failure to induce CD42 expression or polyploidization (Figure 4, A and B). Importantly, JAK2 V617F and c-MPL W515L enhanced phosphorylation of STAT5 and STAT3 but not STAT1, although both of them slightly increased STAT1 expression (Figure 4, C and D). This result shows that without GATA-1, enhanced TPO signaling is insufficient for megakaryocytic maturation. Moreover, STAT1 signaling is uniquely required for megakaryocytic differentiation, whereas STAT3 and STAT5 may be more important in promoting megakaryocyte progenitor survival and proliferation.

IFN- $\gamma$ promotes polyploidization and differentiation of primary bone marrow-derived $\mathrm{CD} 1^{+}$cells independent of TPO. STAT1 is physiologi- 
A

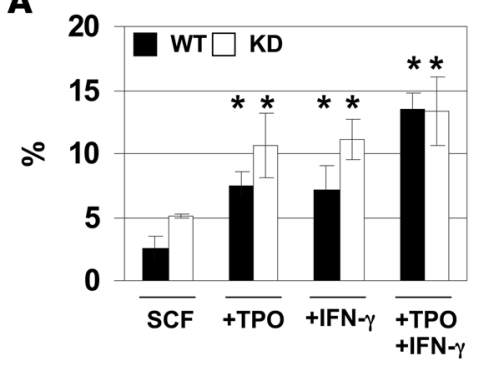

C

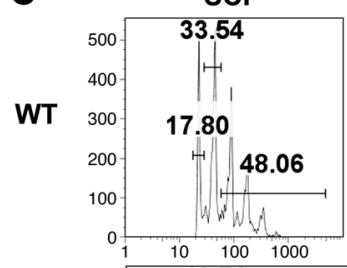

B
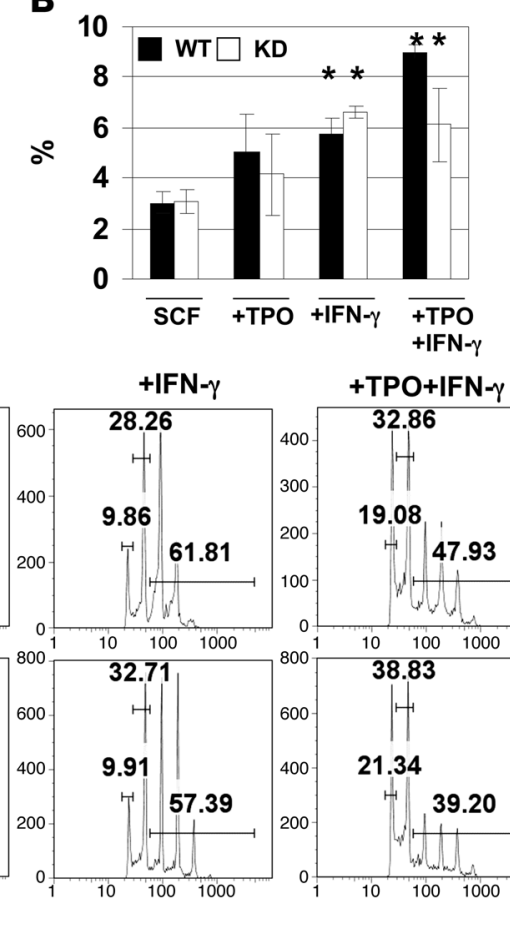

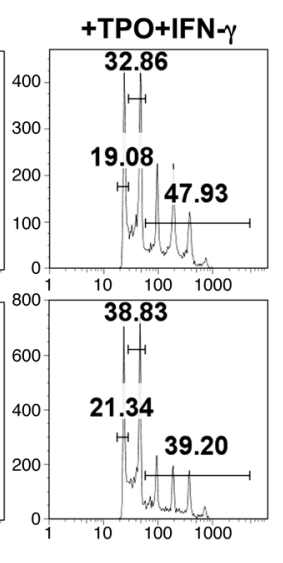

+ TPO
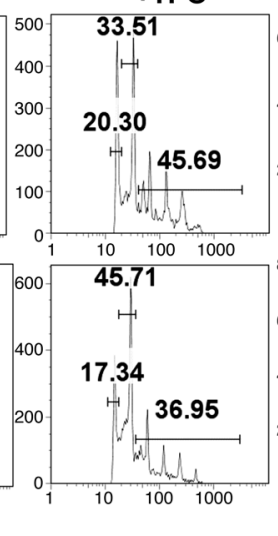

\section{Figure 5}

IFN- $\gamma$ promotes polyploidization in primary megakaryocytes. WT and Gata1-KD (KD) bone marrow cells, harvested from 5-fluorouracil-pretreated mice were cultured in vitro in the presence of SCF alone or SCF plus TPO (+TPO; $20 \mathrm{ng} / \mathrm{ml})$, SCF plus IFN- $\gamma(+\mathrm{IFN}-\gamma$; $20 \mathrm{ng} / \mathrm{ml}$ ), or SCF plus TPO and IFN- $\gamma$ $(+\mathrm{TPO}+\mathrm{IFN}-\gamma)$ as indicated. After 3 days, cells were stained with FITC-labeled anti-CD41, PE-labeled anti-CD42, and DAPI and then analyzed by flow cytometry. CD41 expression (A), CD42 expression (B), and DNA content (C) are shown for each group. Data are representative of 3 independent experiments with similar results. ${ }^{*} P<0.05$ compared with SCF alone group. cally activated by IFN- $\gamma$. To assay the function of STAT1 in megakaryocyte development, we cultured mouse bone marrow progenitors in medium supplemented with SCF and IFN- $\gamma$ but not TPO. In both WT and Gata1-KD progenitors, IFN- $\gamma$ effectively promoted the development of $\mathrm{CD} 41^{+}$and $\mathrm{CD} 42^{+}$cells in the absence of TPO to a similar extent as TPO alone (Figure 5, A and B). Furthermore, we observed a striking reproducible increase in the DNA content of CD $41^{+}$cells cultured in the presence of IFN- $\gamma$ (Figure 5C). These findings are consistent with the increased expression of Ccnd1, Cond2, and Cone1 upon STAT1 activation (Figure 2B) and suggest that activation of STAT 1 by IFN- $\gamma$ can bypass the requirement for GATA-1 in polyploidization. Of note, we observed an additive effect between IFN- $\gamma$ and TPO in inducing expression of CD41 and CD42 but not polyploidization in WT progenitor cells (Figure 5).

STAT1 signaling promotes the early stage of megakaryocyte development and differentiation. To further dissect the role of STAT1 in megakaryocyte development and differentiation, we transduced primary bone marrow cells with GATA-1, STAT1, IRF-1, or GFP alone. After infection, cells were cultured for 4 days in TPO and then evaluated for their state of maturation by flow cytometry. As in G1ME cells, ectopic expression of STAT1 or IRF-1, compared with GFP alone, induced CD41 expression in Gata1-KD bone marrow progenitors (Figure 6, A and B). However, in both cases the extent of CD42 induction was much lower than that in cells expressing GATA-1. STAT1 expression appeared to induce polyploidization of both WT and Gata1-KD megakaryocytes. Indeed, the level of polyploidization was similar in STAT1- and GATA-1-transduced cells (Figure 6C). These data confirm that STAT1 rescues polyploidization and at least partially restores CD41 expression in Gata1-deficient megakaryocytes. Expression of IRF-1 in Gata1-KD progenitors led to intermediate increase in CD41- and CD42-positive cells but only a modest increase in the DNA content (Figure 6), suggesting that additional STAT1 targets participate in megakaryocyte maturation.
Proplatelet formation occurs late in megakaryocyte maturation and can be observed in cultured cells. We detected proplatelets in WT megakaryocytes on day 4 of culture (data not shown). In contrast, we never observed proplatelet formation in Gata1-KD megakaryocytes, consistent with the known requirement for GATA-1 for terminal megakaryocyte differentiation. As expected, overexpression of GATA-1 in Gata1-KD cultures rescued proplatelet formation and also accelerated their development in WT cells, so that in both cases proplatelets could be detected by day 3 (Figure 6, E and I). Overexpression of STAT1 or IRF1, however, did not accelerate maturation in WT cells or rescue proplatelet formation in Gata1KD cells (Figure 6, F, G, J, and K). These results are consistent with our findings in G1ME cells (Figure 1) and suggest that increased STAT1 signaling only partially compensates for loss of GATA-1. Indeed, STAT1 appears to primarily affect polyploidization of megakaryocytes and is not sufficient to rescue platelet biogenesis.

STAT1 deficiency reduces polyploidization in megakaryocytes. To further define the requirements for STAT1 signaling in megakaryocyte differentiation, we studied Stat1 ${ }^{-/-}$mice. A previous study demonstrated normal platelet counts in these mice, but the megakaryocyte lineage was not analyzed in detail (38). Indeed, mutant CD $41^{+}$cells derived from Stat $1^{-/-}$mice bone marrow progenitor cells exhibited significantly reduced DNA content compared with CD $41^{+}$cells from WT littermate controls, confirming that STAT1 signaling contributes to megakaryopoiesis by promoting polyploidization (Figure 7). As further evidence for a specific requirement for STAT1 in polyploidization, CFU-Mk numbers were similar in WT and Stat1 $1^{-/}$mice (Supplemental Figure 4).

\section{Discussion}

Interferons exert multiple biological effects and are generally considered to be negative regulators of cellular proliferation and maturation. Indeed, studies have demonstrated that IFN- $\alpha$ inhibits 
A

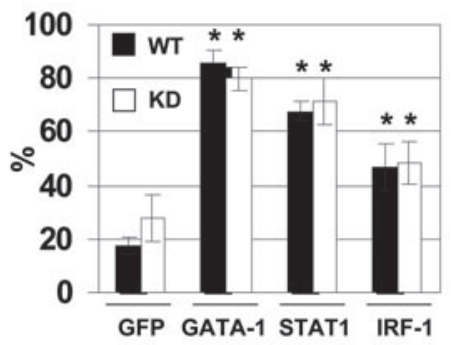

C

GFP
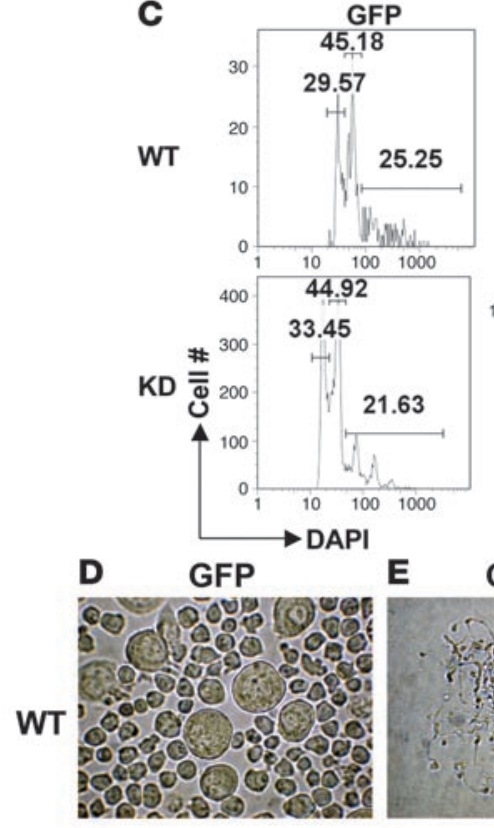

E GATA-1

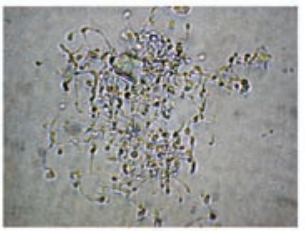

I

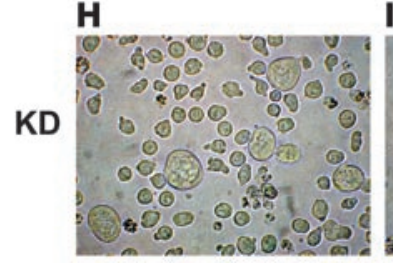

B

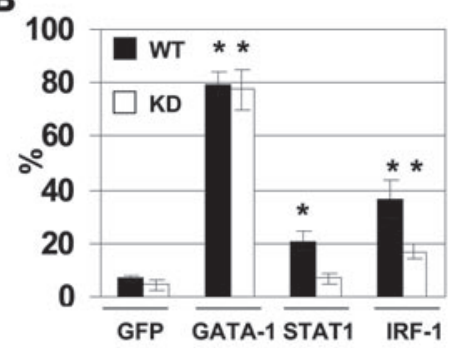

STAT1
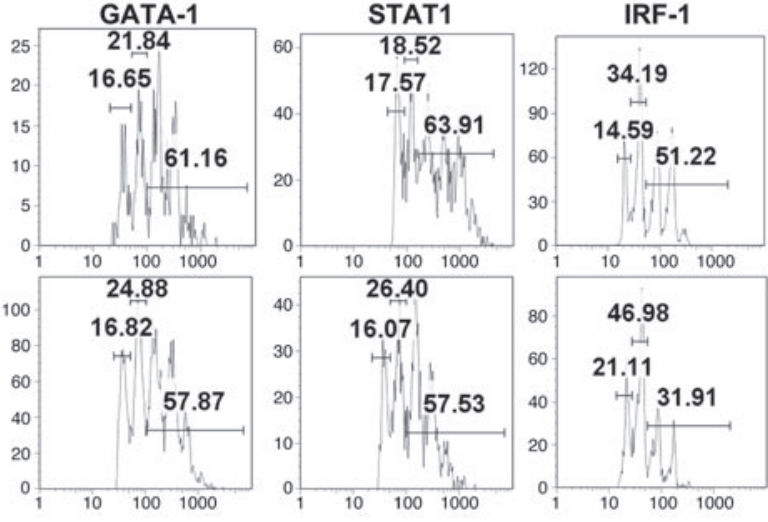

F

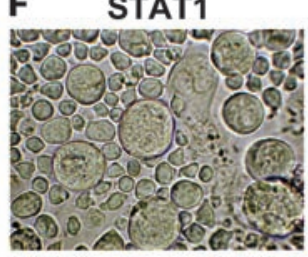

J

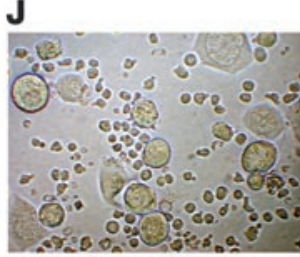

G IRF-1

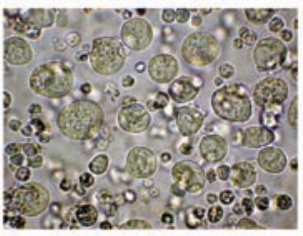

K

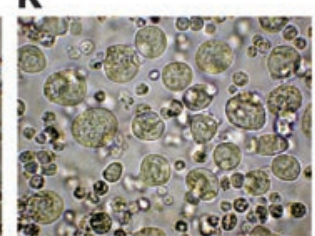

\section{Figure 6}

STAT1 signaling promotes primary megakaryocyte development by facilitating polyploidization. Bone marrow cells from 5-fluorouracil-pretreated WT and Gata1-KD mice were transduced with GFP alone, GATA-1, STAT1, or IRF-1. Transduced cells were purified by sorting and seeded in megakaryocyte differentiation medium (in the presence of $20 \mathrm{ng} / \mathrm{ml}$ TPO) for 3 days. Cells were collected, fixed, permeabilized, stained, and analyzed by flow cytometry. CD41 expression (A), CD42 expression (B), and DNA content $(\mathbf{C})$ of $\mathrm{CD} 41^{+}$cells are shown. (D-K) The morphology and proplatelet formation of transduced bone marrow cells were assessed by light microscopy after 3 days of culture in differentiation medium. Data are representative of 3 independent experiments with similar results. ${ }^{*} P<0.05$ compared with GFP alone group. proliferation of hematopoietic progenitors and suppresses CFU-E, GM, and Mk activities (38-40). Similarly, IFN- $\gamma$, which is known to induce an antiviral state, suppresses the growth of hematopoietic progenitor cells in general (40). Surprisingly, IFN- $\gamma$ supports megakaryopoiesis. Although one study from the 1980s reported that IFN- $\gamma$ inhibited megakaryocyte colony formation in a manner similar to IFN- $\alpha$ (41), subsequent reports have found that IFN- $\gamma$ actually promotes the development of CFU-Mk. For example, IFN- $\gamma$ was found to stimulate megakaryocyte growth under some conditions in vitro (42). In the same way, a marked increase in megakaryocyte numbers was observed in cultures of murine bone marrow cells supplemented with IFN- $\gamma(23)$. These latter findings were recapitulated in vivo, as administration of IFN- $\gamma$, in conjunction with IL-3, facilitated the recovery of platelets and shortened the duration of thrombocytopenia in animals treated with 5-fluorouracil, whereas IL-3 alone had no effect (22). IFN- $\gamma$ also induced megakaryocyte proliferation in bone marrow cultures in combination with SCF (43). Further, when added to cultures containing TPO or SCF, IFN- $\gamma$ significantly augmented the development of megakaryocyte colonies and increased the DNA content of cultured megakaryocytes (24). IFN- $\gamma$ was also found to support TPO-independent proliferation and differentiation of a human megakaryocytic cell line, HIMeg-01 (44). Taken together, the previous findings indicate that IFN- $\gamma$ plays a unique role as a positive regulator of the megakaryocyte lineage.

In this study, we demonstrate that IFN- $\gamma$ and the downstream signaling molecules STAT1 and IRF-1 contribute to megakaryopoiesis by promoting polyploidization and inducing expression of a subset of megakaryocyte-specific genes (Figure 8). This effect is downstream of GATA-1, as Gata1-KD megakaryocytes exhibit reduced levels of STAT1 concomitant with reduced polyploidization and defective maturation, and their polyploidization can be rescued by incubation with IFN- $\gamma$ or ectopic expression of STAT1 or IRF-1. Our work thus sheds light on the molecular mechanism of megakaryopoiesis.

The ability of STAT1 and IRF-1 to promote polyploidization is somewhat surprising, as IFN- $\gamma /$ STAT1 restricts cell growth in most cell types by causing $\mathrm{G}_{1}$ phase arrest and apoptosis through upregulation of p21 and p27 and downregulation of c-Myc (27). In contrast, we found that STAT1- or IRF-1-transduced mega- 

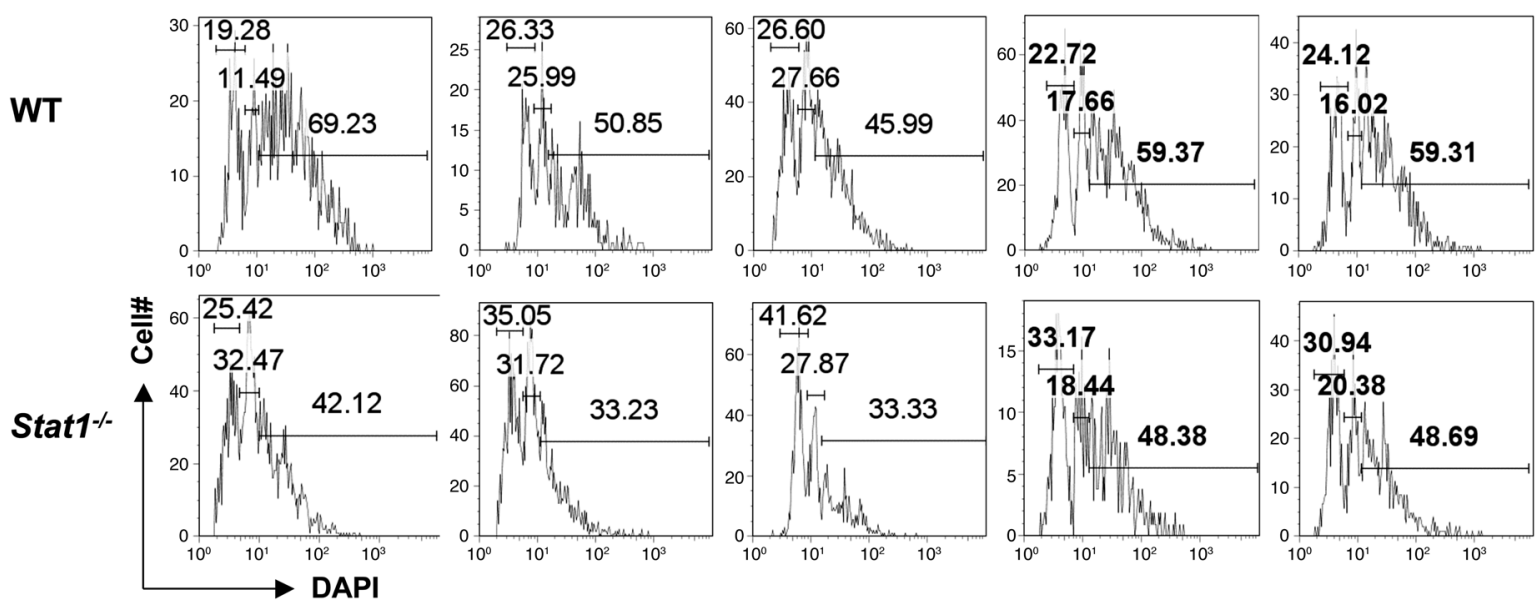

Figure 7

STAT1 deficiency reduces polyploidization of megakaryocytes. Bone marrow progenitor cells from WT and Stat $1^{-/-}$mice were cultured for 3 days to generate megakaryocytes. The resultant cells were stained with FITC-labeled anti-CD41 and DAPI and analyzed for DNA content by flow cytometry. Statistical analysis from 5 pairs of WT and Stat1-1- mice showed that the percentage of megakaryocytes with high polyploidy (equal to or greater than $8 \mathrm{~N}$ ) was significantly different in the 2 groups $\left(57.0 \pm 8.9\right.$ for WT and $41.1 \pm 7.7$ for Stat ${ }^{-1}($ mean \pm SD; $n=5 ; P=0.017$, unpaired Student's $t$ test).

karyocytes showed elevated expression of Cond1, Ccnd2, and Ccne1, all of which contribute to endomitosis of megakaryocytes (30-33). Similarly, IFN- $\gamma$ also increased the DNA content of WT and GATA-1-deficient megakaryocytes. Thus, IFN- $\gamma$ signaling likely promotes polyploidization by increasing the expression of $\mathrm{G}_{1}$ cyclins. Of note, we did not observe significant changes in the mRNA expression of other cell-cycle factors proposed to play a role in megakaryopoiesis, including Ccna1, Ccnb1, Cdc2, p21, p27, Survivin, and Aim1, consistent with a recent study of gene expression profiling during megakaryocyte maturation (45). Our data do not exclude the possibility that IFN- $\gamma$ signaling regulates these genes at the protein level.

In addition to having a reduced state of polyploidization, Gata1-deficient megakaryocytes are defective in platelet production. We found that while expression of STAT1 and IRF-1 led to increased DNA content and increased expression of a subset of late megakaryocyte genes, such as $N f e 2$, platelet synthesis was not restored. This suggests that STAT1 contributes to transcription of a subset of GATA-1 target genes but cannot rescue full differentiation. In contrast to the differentiation effects of STAT1 and IRF-1, expression of constitutively active JAK2 or c-MPL failed to promote megakaryocytic differentiation of Gata1-null hematopoietic cells. Rather, these factors promoted proliferation and TPO-independent growth exclusively, without affecting maturation. This finding is consistent with observations that mutations in JAK2 contribute to greater than $90 \%$ of cases of polycythemia vera (PV) and nearly $50 \%$ of cases of essential thrombocythemia and PMF, while $c-M P L$ W515L mutation contributes to approximately $10 \%$ of PMF cases (37). Most likely, enhanced JAK2 signaling cannot overcome the Gata1 deficien$\mathrm{cy}$, as it does not activate STAT1 and subsequent expression of essential downstream differentiation targets. These observations suggest that activation of JAK signaling coupled with a differentiation block might promote leukemia. Indeed, recent studies demonstrated that mutations in JAK2 and JAK3 are associated with AMKL (46-48). Our current studies raise the possibility that activation of STAT1 through IFN- $\gamma$ or other compounds might serve as a novel differentiation therapy for AMKL.

It is also important to consider whether IFN- $\gamma$ could provide any therapeutic benefit for patients with PMF. Among the MPDs, PMF patients uniquely harbor $c-M P L$ mutations (37) and also show significantly reduced expression levels of GATA-1 (6). Mice with reduced GATA-1 expression develop a disease that resembles human PMF, characterized by myelofibrosis, extramedullary hematopoiesis, poikilocytosis, and anemia (5). In addition, the mutant bone marrow expresses higher levels of growth factors associated with myelofibrosis, including TGF- $\beta 1$, PDGF, and VEGF. With respect to the megakaryocyte lineage, the observed maturation defects in the mouse model and human patients with PMF are identical. Given that IFN- $\gamma$ promoted the polyploidization of GATA-1-KD progenitors and GATA-1-null G1ME cells, it is possible that this cytokine might ameliorate at least some of the features of PMF. However, the GATA-1 deficiency cannot be fully compensated by IFN- $\gamma$ or ectopic expression of STAT1, so it remains unclear to what extent IFN- $\gamma$ might benefit PMF patients.

Although Stat $1^{-/-}$mice do not show significant alteration in platelet numbers, their megakaryocytes reach a lower ploidy state compared with those of their WT littermates. This observation is similar to the finding that despite not having obvious changes in circulating erythrocytes, Stat $1^{-/}$mice display altered erythropoiesis, which is characterized by an overall reduction in erythroid progenitors, a less differentiated phenotype, and increased apoptosis of early erythroblasts (38). Thus, in both lineages, STAT1 plays a subtle but meaningful role that may become more profound under certain hematopoietic stresses. The absence of a more profound defect in these lineages may be the consequence of continued expression of STAT3 and STAT5.

Platelet counts are normally maintained in a physiologic range through a variety of homeostatic mechanisms. Elevated platelet counts occur via both cell-autonomous and non-cell-autonomous mechanisms. Essential thrombocythemia is caused by 


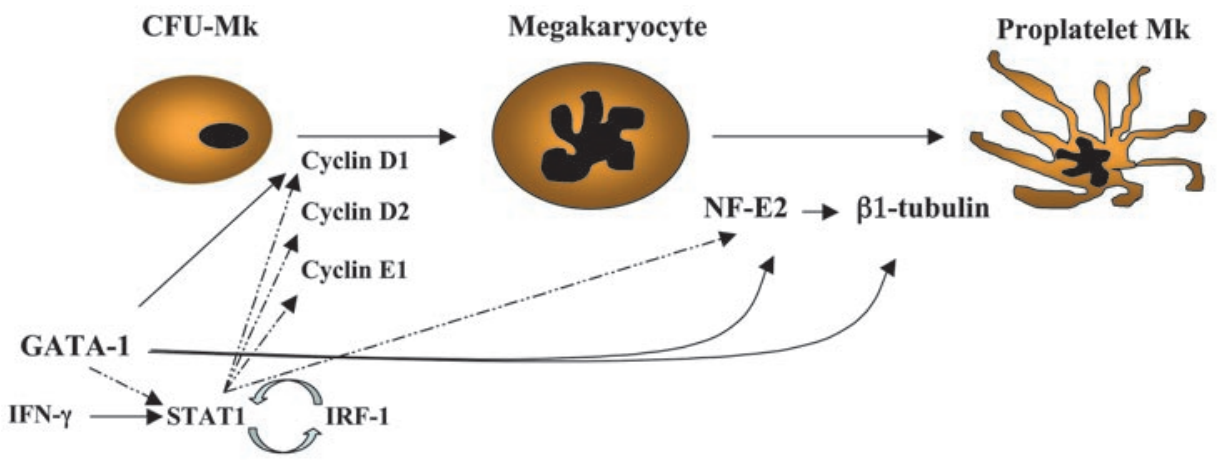

\begin{abstract}
Figure 8
Model of the STAT1/GATA-1 axis in megakaryopoiesis. STAT1 activation, mediated by GATA- 1 and IFN- $\gamma$ signaling, contributes to megakaryopoiesis primarily by facilitating expression of multiple endomitotic regulators, including cyclins D1 and D2. STAT1 also likely acts downstream of GATA-1 to promote expression of other key regulators, such as NF-E2.
\end{abstract}

hematopoietic cell-intrinsic defects, frequently acquired mutations in JAK2. More commonly, thrombocytosis is a secondary, reactive condition. In this latter case, increased platelet counts are driven by inappropriate cytokine production during infection, cancer, and chronic inflammation (49). Common mediators of reactive thrombocytosis include IL-1 $\beta$, IL-6, GM-CSF, TNF- $\alpha$, and IFN- $\gamma$ (50). Recently, stromal cell-derived factor 1 (SDF-1) has been shown to promote megakaryopoiesis and thrombopoiesis $(51,52)$. However, in our liquid culture system, we did not observe any effect of SDF- 1 in promoting $\mathrm{CD} 41^{+}$and $\mathrm{CD} 42^{+}$cells compared with TPO or IFN- $\gamma$ groups (Supplemental Figure 5). Consistent with previous observations, we also failed to detect any STAT1 phosphorylation activated by SDF-1 stimulation in WT BM cells or a mouse megakaryocytic cell line, Y10, both of which express SDF-1 receptor CXCR4 (53). We note that our experiments do not exclude a function for SDF- 1 in promoting other aspects of thrombopoiesis. Taken together, our results provide new insights into the unique actions of IFN- $\gamma$ and its downstream target STAT1 in megakaryopoiesis. Specifically, we propose that STAT1 is a critical effector of GATA-1 during normal megakaryocyte development and that inappropriate activation of this pathway by IFN- $\gamma$ during various pathologic states directly contributes to thrombocytosis by enhancing megakaryocyte maturation. This provides one mechanism by which inflammatory disorders predispose affected individuals to pathologic thrombosis.

\section{Methods}

Hematopoietic cells and megakaryocyte culture. G1ME cells were maintained in $\alpha$-MEM media supplemented with $20 \%$ FBS and $1 \%$ TPO conditioned medium as described previously (24). To culture primary megakaryocytes, mice were treated with 5 -fluorouracil ( $100 \mathrm{mg} / \mathrm{kg}$ mice body) by intraperitoneal injection, and 5 days later, the progenitor cells were harvested by flushing bone marrow of tibia and fibula. Alternatively, progenitor cells were enriched from bone marrow of untreated mice by negative selection using a progenitor cell enrichment kit (Stem Cell Technologies). Megakaryocytes were cultured in a serum-free system as described previously (4). C57BL/6 and GATA-1-KD mice were maintained in microisolator housing within a barrier facility. WT and Stat $1^{-/}$littermates were maintained on BALB/c genetic background. All animal studies were approved by the Animal Care and Use Committees of Northwestern University, The University of Chicago, and the Ontario Cancer Institute.

Retroviral transduction. The virus-packaging cells Plat-E $\left(5 \times 10^{6}\right)$ were seeded in a $10-\mathrm{cm}$ dish the day before transfection and then transfected with MSCV-based retroviral vector MIGR1 containing GATA-1, STAT1, or IRF-1 cDNA using Lipofectamine 2000 (Invitrogen) following the manu- facturer's protocols. Forty-eight hours after transfection, the viral supernatant was collected, and $5 \mathrm{ml}$ of viral supernatant was mixed with $4 \times 10^{6}$ of G1ME cells or bone marrow progenitor cells in the presence of $8 \mu \mathrm{g} / \mathrm{ml}$ polybrene and $10 \mathrm{mM}$ HEPES and spun at $2,300 \mathrm{~g}$ for 1.5 hours at room temperature. Spinoculation was performed 4 times over 2 days.

Intracellular staining. Cells were first fixed by incubation with $2 \%$ paraformaldehyde at room temperature for 10 minutes, and then, after washing, they were again fixed in $90 \%$ methanol for 30 minutes on ice. After 1 hour incubation on ice in PBS supplemented with $0.1 \%$ BSA, cells were centrifuged and resuspended with $1 \mu \mathrm{l}$ of $2.4 \mathrm{G} 2$ and $20 \mu \mathrm{l}$ of Alexa Fluor 647- or PE-labeled antibodies specific to phospho-STAT1, -STAT3, and -STAT5 (BD Biosciences - Pharmingen), and cells were incubated at room temperature for 1 hour. After addition of $200 \mu \mathrm{l}$ PBS, cells were analyzed by flow cytometry on a BD FACSCanto. Data were analyzed using FlowJo software (Treestar).

Quantitative RT-PCR. GFP-, GATA-1-, STAT1-, or IRF-1-transduced G1ME cells were purified by sorting. Total RNA was isolated from purified cells. cDNA was generated from $1 \mu \mathrm{g}$ of total RNA using reverse transcriptase following the manufacturer's instructions. Real-time PCR was performed in a $20-\mu 1$ reaction. The PCR amplification conditions were: hotstart at $95^{\circ} \mathrm{C}$ for 15 minutes followed by $95^{\circ} \mathrm{C}$ for 30 seconds, $60^{\circ} \mathrm{C}$ for 2 minutes for 40 cycles. The relative quantitation of real-time PCR product was measured using the comparative $\Delta \Delta \mathrm{C}_{\mathrm{T}}$ method (as described in ref. 54 ) and presented in bar graph format. Note the different scales used for different genes due to the huge variation of expression. The means and standard deviations of all qRT-PCR reactions are also provided in Supplemental Table 1. Primer sequences for the following genes were derived from previous publications (4, 24): c-Myc, Gata1, Ccnd1, Ccnd2, Ccnd3, p16, $p 21, p 27$, Gata2. Other primer sequences are provided in Supplemental Materials and Methods.

Western blot analysis. Cells were lysed in RIPA buffer (Tris-HCl, $50 \mathrm{mM}$, pH 7.4; NP-40, 1\%; Na-deoxycholate, $0.25 \%$; NaCl, 150 mM; EDTA, 1 mM) supplemented with protease inhibitors (pepstatin, leupeptin, aprotinin, each $10 \mu \mathrm{g} / \mathrm{ml}$; PMSF, $1 \mathrm{mM}$ ) and phosphatase inhibitor $\left(\mathrm{Na}_{3} \mathrm{Vo}_{4}, 1 \mathrm{mM}\right)$. Protein was separated by SDS-PAGE and transferred to a PVDF membrane. Membranes were blotted with antibodies detecting GATA-1 (N6), IRF-1, STAT3, STAT5, and HSC70 (each from Santa Cruz Biotechnology Inc). STAT1(h) (Santa Cruz) and STAT1(m) (BD Biosciences) antibodies recognize human and mouse STAT1, respectively. Membranes were also blotted with antibodies specifically detecting phosphorylation of STAT1 (p-STAT1), STAT3 (p-STAT3), and STAT5 (p-STAT5) (Santa Cruz Biotechnology Inc.).

Information about luciferase assays and additional primer pair sequences are provided in Supplemental Materials and Methods.

Statistics. All statistical analyses were performed using the Student's $t$ test ( 2 tailed, unpaired). A $P$ value of 0.05 or less was considered significant. 


\section{Acknowledgments}

We thank for Ross Levine and Gary Gilliland for the JAK2 V617F and c-MPL W515L constructs and Sandeep Gurbuxani and members of the Crispino lab for helpful discussions. This study is supported by a grant from the NIH (CA101774) and the Canadian Institute of Health Research (D.L. Barber). J.D. Crispino is a scholar of the Leukemia and Lymphoma Society.

Received for publication June 15, 2007, and accepted in revised form October 1, 2007.

1. Crispino, J.D. 2005. GATA1 in normal and malignant hematopoiesis. Semin. Cell Dev. Biol. 16:137-147.

2. Shivdasani, R.A., Fujiwara, Y., McDevitt, M.A., and Orkin, S.H. 1997. A lineage-selective knockout establishes the critical role of transcription factor GATA-1 in megakaryocyte growth and platelet development. EMBO J. 16:3965-3973.

3. Vyas, P., Ault, K., Jackson, C.W., Orkin, S.H., and Shivdasani, R.A. 1999. Consequences of GATA-1 deficiency in megakaryocytes and platelets. Blood. 93:2867-2875.

4. Muntean, A.G., and Crispino, J.D. 2005. Differential requirements for the activation domain and FOGinteraction surface of GATA-1 in megakaryocyte gene expression and development. Blood. 106:1223-1231.

5. Vannucchi, A.M., et al. 2002. Development of myelofibrosis in mice genetically impaired for GATA-1 expression (GATA-1(low) mice). Blood. 100:1123-1132.

6. Vannucchi, A.M., et al. 2005. Abnormalities of GATA-1 in megakaryocytes from patients with idiopathic myelofibrosis. Am. J. Pathol. 167:849-858.

7. Wechsler, J., et al. 2002. Acquired mutations in GATA1 in the megakaryoblastic leukemia of Down syndrome. Nat. Genet. 32:148-152.

8. Li, Z., et al. 2005. Developmental stage-selective effect of somatically mutated leukemogenic transcription factor GATA1. Nat. Genet. 37:613-619.

9. Kaushansky, K. 2005. The molecular mechanisms that control thrombopoiesis. J. Clin. Invest. 115:3339-3347.

10. Sattler, M., et al. 1995. The thrombopoietin receptor c-MPL activates JAK2 and TYK2 tyrosine kinases. Exp. Hematol. 23:1040-1048.

11. Drachman, J.G., Sabath, D.F., Fox, N.E., and Kaushansky, K. 1997. Thrombopoietin signal transduction in purified murine megakaryocytes. Blood. 89:483-492.

12. Kaushansky, K., and Drachman, J.G. 2002. The molecular and cellular biology of thrombopoietin: the primary regulator of platelet production. Oncogene. 21:3359-3367.

13. Kirito, K., and Kaushansky, K. 2006. Transcriptional regulation of megakaryopoiesis: thrombopoietin signaling and nuclear factors. Curr. Opin. Hematol. 13:151-156.

14. Neubauer, H., et al. 1998. Jak2 deficiency defines an essential developmental checkpoint in definitive hematopoiesis. Cell. 93:397-409.

15. Parganas, E., et al. 1998. Jak2 is essential for signaling through a variety of cytokine receptors. Cell. 93:385-395.

16. Benekli, M., Baer, M.R., Baumann, H., and Wetzler, M. 2003. Signal transducer and activator of transcription proteins in leukemias. Blood. 101:2940-2954.

17. Steensma, D.P., et al. 2006. JAK2 V617F is a rare finding in de novo acute myeloid leukemia, but STAT3 activation is common and remains unexplained. Leukemia. 20:971-978.

18. Snow, J.W., et al. 2002. STAT5 promotes multilineage hematolymphoid development in vivo through effects on early hematopoietic progenitor cells. Blood. 99:95-101.

19. Kirito, K., et al. 2002. A functional role of Stat3 in in vivo megakaryopoiesis. Blood. 99:3220-3227.

Address correspondence to: John D. Crispino, Northwestern University Feinberg School of Medicine, Division of Hematology and Oncology, 303 E. Superior Street, Lurie Research Building 5-113, Chicago, Illinois 60611, USA. Phone: (312) 503-1504; Fax: (312) 503-0189; E-mail: j-crispino@northwestern.edu.

Andrew G. Muntean's present address is: Department of Pathology, University of Michigan Health System, Ann Arbor, Michigan, USA.

20. Ezoe, S., et al. 2005. GATA transcription factors inhibit cytokine-dependent growth and survival of a hematopoietic cell line through the inhibition of STAT3 activity. J. Biol. Chem. 280:13163-13170.

21. Kisseleva, T., Bhattacharya, S., Braunstein, J., and Schindler, C.W. 2002. Signaling through the JAK/ STAT pathway, recent advances and future challenges. Gene. 285:1-24.

22. Tsuji-Takayama, K., et al. 1996. IFN-gamma in combination with IL-3 accelerates platelet recovery in mice with 5 -fluorouracil-induced marrow aplasia. J. Interferon Cytokine Res. 16:447-451.

23. Tsuji-Takayama, K., et al. 1996. Interferon-gamma enhances megakaryocyte colony-stimulating activity in murine bone marrow cells. J. Interferon Cytokine Res. 16:701-708.

24. Muraoka, K., et al. 1997. Thrombopoietin-independent effect of interferon-gamma on the proliferation of human megakaryocyte progenitors. $\mathrm{Br}$. J. Haematol. 98:265-273.

25. Stachura, D.L., Chou, S.T., and Weiss, M.J. 2006. Early block to erythromegakaryocytic development conferred by loss of transcription factor GATA- 1 . Blood. 107:87-97.

26. Schroder, K., Hertzog, P.J., Ravasi, T., and Hume, D.A. 2004. Interferon-gamma: an overview of signals, mechanisms and functions. J. Leukoc. Biol. 75:163-189.

27. Schulze, H., and Shivdasani, R.A. 2005. Mechanisms of thrombopoiesis. J. Thromb. Haemost. 3:1717-1724.

28. Ichikawa, M., et al. 2004. AML-1 is required for megakaryocytic maturation and lymphocytic differentiation, but not for maintenance of hematopoietic stem cells in adult hematopoiesis. Nat. Med. 10:299-304.

29. Elagib, K.E., et al. 2003. RUNX1 and GATA-1 coexpression and cooperation in megakaryocytic differentiation. Blood. 101:4333-4341.

30. Muntean, A.G., et al. 2007. Cyclin D-Cdk4 is regulated by GATA-1 and required for megakaryocyte growth and polyploidization. Blood. 109:5199-5207.

31. Wang, Z., Zhang, Y., Kamen, D., Lees, E., and Ravid, K. 1995. Cyclin D3 is essential for megakaryocytopoiesis. Blood. 86:3783-3788.

32. Matsumura, I., et al. 2000. Increased D-type cyclin expression together with decreased cdc2 activity confers megakaryocytic differentiation of a human thrombopoietin-dependent hematopoietic cell line. J. Biol. Chem. 275:5553-5559.

33. Geng, Y., et al. 2003. Cyclin E ablation in the mouse. Cell. 114:431-443.

34. De Botton, S., et al. 2002. Platelet formation is the consequence of caspase activation within megakaryocytes. Blood. 100:1310-1317.

35. Ogilvy, S., et al. 1999. Constitutive Bcl-2 expression throughout the hematopoietic compartment affects multiple lineages and enhances progenitor cell survival. Proc. Natl. Acad. Sci. U. S. A. 96:14943-14948.

36. Kaluzhny, Y., et al. 2002. BclxL overexpression in megakaryocytes leads to impaired platelet fragmentation. Blood. 100:1670-1678.

37. Levine, R.L., Pardanani, A., Tefferi, A., and Gilliland, D.G. 2007. Role of JAK2 in the pathogenesis and therapy of myeloproliferative disorders. Nat. Rev. Cancer. 7:673-683.

38. Halupa, A., et al. 2005. A novel role for STAT1 in regulating murine erythropoiesis: deletion of STAT1 results in overall reduction of erythroid progenitors and alters their distribution. Blood. 105:552-561.

39. Wang, Q., Miyakawa, Y., Fox, N., and Kaushansky, K. 2000. Interferon-alpha directly represses megakaryopoiesis by inhibiting thrombopoietininduced signaling through induction of SOCS-1. Blood. 96:2093-2099.

40. Kato, K., et al. 2003. Intracellular signal transduction of interferon on the suppression of haematopoietic progenitor cell growth. Br. J. Haematol. 123:528-535.

41. Ganser, A., Carlo-Stella, C., Greher, J., Volkers, B., and Hoelzer, D. 1987. Effect of recombinant interferons alpha and gamma on human bone marrowderived megakaryocytic progenitor cells. Blood. 70:1173-1179.

42. Griffin, C.G., and Grant, B.W. 1990. Effects of recombinant interferons on human megakaryocyte growth. Exp. Hematol. 18:1013-1018.

43. Shiohara, M., Koike, K., and Nakahata, T. 1993. Synergism of interferon-gamma and stem cell factor on the development of murine hematopoietic progenitors in serum-free culture. Blood. 81:1435-1441.

44. Li, J., et al. 1998. Megakaryocytic differentiation of HIMeg- 1 cells induced by interferon gamma and tumour necrosis factor alpha but not by thrombopoietin. Cytokine. 10:880-889.

45. Raslova, H., et al. 2007. Interrelation between polyploidization and megakaryocyte differentiation: a gene profiling approach. Blood. 109:3225-3234.

46. Jelinek, J., et al. 2005. JAK2 mutation $1849 \mathrm{G}>\mathrm{T}$ is rare in acute leukemias but can be found in CMML, Philadelphia chromosome-negative CML, and megakaryocytic leukemia. Blood. 106:3370-3373.

47. Steensma, D.P. 2006. JAK2 V617F in myeloid disorders: molecular diagnostic techniques and their clinical utility: a paper from the 2005 William Beaumont Hospital Symposium on Molecular Pathology. J. Mol. Diagn. 8:397-411; quiz 526.

48. Walters, D.K., et al. 2006. Activating alleles of JAK3 in acute megakaryoblastic leukemia. Cancer Cell. 10:65-75.

49. Schafer, A.I. 2004. Thrombocytosis. N. Engl. J. Med. 350:1211-1219.

50. Griesshammer, M., et al. 1999. Aetiology and clinical significance of thrombocytosis: analysis of 732 patients with an elevated platelet count. J. Intern. Med. 245:295-300.

51. Hamada, T., et al. 1998. Transendothelial migration of megakaryocytes in response to stromal cellderived factor 1 (SDF-1) enhances platelet formation. J. Exp. Med. 188:539-548.

52. Hodohara, K., Fujii, N., Yamamoto, N., and Kaushansky, K. 2000. Stromal cell-derived factor1 (SDF-1) acts together with thrombopoietin to enhance the development of megakaryocytic progenitor cells (CFU-MK). Blood. 95:769-775.

53. Majka, M., et al. 2000. Stromal-derived factor 1 and thrombopoietin regulate distinct aspects of human megakaryopoiesis. Blood. 96:4142-4151.

54. Applied Biosystems. 1997. User Bulletin no. 2: ABI PRISM 7700 Sequence Detection System. docs. appliedbiosystems.com/pebiodocs/04303859.pdf. 